\title{
DNA Translocation in Inorganic Nanotubes
}

\author{
Rong Fan ${ }^{1^{*}}$, Rohit Karnik ${ }^{2 *}$, Min Yue ${ }^{2}$, Deyu $\mathrm{Li}^{2}$, Arun Majumdar ${ }^{2,3, \dagger}$, Peidong Yang ${ }^{1,3, \dagger}$ \\ ${ }^{1}$ Department of Chemistry, University of California, Berkeley, CA 94720, USA \\ ${ }^{2}$ Department of Mechanical Engineering, University of California, Berkeley, CA 94720, \\ USA \\ ${ }^{3}$ Materials Sciences Division, Lawrence Berkeley National Laboratory, Berkeley, CA \\ 94720, USA
}

\section{Fabrication of Nanotube Nanofluidic Devices}

Inorganic nanotube nanofluidic devices were fabricated in two steps --- chemical synthesis of core-sheath $\mathrm{Si} / \mathrm{SiO}_{2}$ nanowires for nanofluidic channels and subsequent integration with lithographically defined microfluidic channels. Silicon nanowires were synthesized ${ }^{1}$ and then oxidized in dry $\mathrm{O}_{2}$ at $850{ }^{\circ} \mathrm{C}$ for 1 hour to form $\mathrm{Si} / \mathrm{SiO}_{2}$ core-sheath nanowires with a $35 \mathrm{~nm}$ silica sheath. After dispersion of the $\mathrm{Si} / \mathrm{SiO}_{2}$ nanowires onto fused silica substrates, a $100 \mathrm{~nm} \mathrm{Cr}$ metal layer was sputtered and lithographically patterned to form a $3 \mu \mathrm{m}$ wide $\mathrm{Cr}$ line on the $\mathrm{Si} / \mathrm{SiO}_{2}$ nanowire serving as an alignment mark for defining the microfluidic channels. $2 \mu \mathrm{m}$ thick low temperature oxide was then deposited on the entire substrate using low-pressure chemical vapor deposition (LPCVD) with $\mathrm{SiH}_{4}$ chemistry and then densified by annealing in inert gas ambient. Two microfluidic channels are patterned and etched to connect both ends of the $\mathrm{Si} / \mathrm{SiO}_{2}$ nanowires. Finally the silicon core of the nanowire was etched away using $\mathrm{XeF}_{2}$ to form the silica nanotube and the resulting devices were plasma bonded with a PDMS cover in which access holes had already been drilled. $\mathrm{Ag} / \mathrm{AgCl}$ electrodes were inserted in the access holes for electrical measurements.

\section{Electrical measurements}

All the electric measurements were conducted in a Faraday. Single molecule sensing experiments were conducted using Keithley Model 428 PROG amplifier. The rise time of $33 \mu \mathrm{sec}$ was set for all the experiments. Data collection was carried out with National Instrument DAQ 6052E PCI card (maximum sampling rate 100,000) controlled by Labview program. Keithley 236 source measure unit was used to characterize the basic electrical properties of ion transport in single nanotubes. All the electric measurements were conducted in a non-standard clean room to avoid dust contamination.

\footnotetext{
${ }^{1}$ Fan, R., Wu, Y., Li, D., Yue, M., Majumdar, A. \& Yang P. Fabrication of silica nanotube arrays from vertical silicon nanowire templates. J. Am. Chem. Soc. 125, 5254-5255 (2003).
}

\footnotetext{
* These authors contributed equally to this paper

${ }^{\dagger}$ Corresponding authors: p_yang@berkeley.edu; majumdar@me.berkeley.edu
} 